\title{
THE FIELD OF FORCE DUE TO A MOVING ELECTRON
}

\author{
By A. W. Conway.
}

[Received January 30th, 1903.-Read February 12th, 1903.-Received in rovised form May 6th, 1903.]

THE following paper treats of the field of force due to the motion of an electron. No assumption is made as to the nature or shape of an electron except to regard it as a simple singularity of the differential equations which is changing its position in three-dimensional space in any continuous manner whatever. The results will, however, be correct as long as the distance of the point at which the field of force is calculated is great compared with the dimensions of the electron. In $\S 1$ the case of an electron moving in a general manner with a speed less than that of radiation is considered. In $\$ 2$ the results are extended to moving distributions of electric charge. Without making an hypothesis concerning the distribution of the charge of the electrical nucleus or electron it it is impossible to obtain the total amount of energy of the system, but the amount of energy radiated, or "wasted," is calculated in $\$ 3$. In $\S 4$ the form which the results of $\S 1$ assume when the speed of the singularity is greater than that of radiation is considered.

\section{The Electromagnetic Field due to an Electron moving with a Velocity less than that of Radiation.}

The differential equations which hold in any space free from singulariarities are, the origin being fixed in the æther,

$$
K \frac{\partial}{\partial t}(X, Y, Z)=\operatorname{curl}(a, \beta, \gamma), \quad-\mu \frac{\partial}{\partial t}(\alpha, \beta, \gamma)=\operatorname{curl}(X, Y, Z)
$$

where $(X, Y, Z)$ is the electric force in electromagnetic units, $(\alpha, \beta, \gamma)$ is the magnetic force, $K$ is the specific inductive capacity, and $\mu$ is the magnetic permeability.

From the above equations it follows that $(X, Y, Z)$ is circuital and that $X, Y$, and $Z$ are solutions of $\nabla^{2} U=V^{-2} \partial^{2} U / \partial t^{2}$ where $V^{-2}=K \mu$.

Let $f_{1}(u), f_{2}(u), f_{3}(u)$ be any functions of $u$ which are real and uniform where $u$ is real, and which are finite and continuous for all finite 
values of $u$ real or complex. Further, let us suppose that for all real positive values of $u$ less than a positive quantity $t$ the condition

$$
\left[f_{1}^{\prime}(u)\right]^{2}+\left[f_{2}^{\prime}(u)\right]^{2}+\left[f_{3}^{\prime}(u)\right]^{2}<V^{2}
$$

is satisfied where $f_{1}^{\prime}(u)$ denotes $\partial f_{1}(u) / \partial u, \ldots$ This condition, as we shall see later, makes the velocity of the electron less than the velocity of radiation.

Consider first points which lie inside the sphere

$$
\left[x-f_{1}(0)\right]^{2}+\left[y-f_{2}(0)\right]^{2}+\left[z-f_{3}(0)\right]^{2}=V^{2} t^{2} .
$$

The function

$$
\phi(u)=\left[x-f_{1}(u)\right]^{2}+\left[y-f_{2}(u)\right]^{2}+\left[z-f_{3}(u)\right]^{2}-V^{2}(t-u)^{2}
$$

has for its first derived

$$
\phi^{\prime}(u)=-2 f_{1}^{\prime}(u)\left[x-f_{1}(u)\right]-2 f_{2}^{\prime}(u)\left[y-f_{2}(u)\right]-2 f_{3}^{\prime}(u)\left[z-f_{3}(u)\right]+2 V^{2}(t-u) .
$$

Within the range $t>u>0$ of real values of $u, \phi^{\prime}(u)$ may have no zero or it may have one or more zeroes. If it has no zero, then, since $\phi(t)$ is positive (being a sum of real squares) and $\phi(0)$ is negative $[(x, y, z)$ lying inside the sphere $\phi(0)=0$ ], it follows that $\phi(u)$ has one, and only one, zero in the range considered. If $\phi^{\prime}(u)$ has one or more zeroes, let $a$ be one of them, so that $\phi^{\prime}(a)=0$. Making use of this, we can write $\phi(a)$ in the form

$$
\begin{aligned}
{\left[x-f_{1}(a)\right]^{2}+} & {\left[y-f_{2}(a)\right]^{2}+\left[z-f_{3}(a)\right]^{2} } \\
& -V^{-2}\left\{\left[x-f_{1}(a)\right] f_{1}^{\prime}(a)+\left[y-f_{2}(a)\right] f_{: !}^{\prime \prime}(a)+\left[z-f_{3}(a)\right] f_{3}^{\prime}(a)\right)^{\prime 2} .
\end{aligned}
$$

If we put

$$
\begin{aligned}
& \xi=l_{1}\left[x-f_{1}(a)\right]+m_{1}\left[y-f_{2}(a)\right]+n_{1}\left[z-f_{3}(a)\right] . \\
& \eta=l_{2}\left[x-f_{1}(a)\right]+m_{2}\left[y-f_{2}(a)\right]+n_{2}\left[z-f_{3}(a)\right] . \\
& \xi=l_{3}\left[x-f_{1}(a)\right]+m_{3}\left[y-f_{2}(a)\right]+n_{3}\left[z-f_{3}(a)\right],
\end{aligned}
$$

where the transformation is real and orthogonal and

$$
l_{1}: m_{1}: n_{1}=f_{1}^{\prime}(a): f_{2}^{\prime}(a): f_{3}^{\prime}(a),
$$

then $\quad \phi(a)=\xi^{2}\left(1-V^{-2}\left\{\left[f_{1}^{\prime}(a)\right]^{2}+\left[f_{2}^{\prime}(a)\right]^{2}+\left[f_{3}^{\prime}(a)\right]^{2}\right\}\right)+\eta^{2}+\xi^{2}$;

so that $\phi(a)$ is positive, unless $\xi=0, \eta=0, \xi=0$. As before, it follows that $\phi(u)$ has only one zero in the range $t>u>0$. It also is evident that, if $\phi(a)=0$ and $\phi^{\prime}(a)=0$, we must have $\xi=0, \eta=0, \xi=0$. These conditions lead to $x=f_{1}(a), y=f_{2}(a), z=f_{3}(a)$; and, since $\phi(a)=0$, we get in this case $t=a$. Summing up then, we see that $\phi(u)$ has one, and only one, zero, $T$ (say), between 0 and $t$, and that $\phi^{\prime}(T$ ) is not zero 
unless $x=f_{1}(t), y=f_{2}(t), z=f_{8}(t)$. In this case $T$ is a simple zero of $\phi^{\prime}(T)$ and a double zero of $\phi(T)$.

The equation $\nabla^{2} U=V^{-2} \partial^{2} U / \partial t^{2}$ is satisfied by

$$
\left\{\left[x-f_{1}(u)\right]^{2}+\left[y-f_{2}(u)\right]^{2}+\left[z-f_{8}(u)\right]^{2}-V^{2}(t-u)^{2}\right\}^{-1}
$$

where $u$ is independent of $x, y, z$, and $t$. It is also satisfied by any number of such solutions provided that the path of summation is independent of $x, y, z$, and $t$. Hence $\int\{\phi(u)\}^{-1} d u$ is a solution where $u$ is a complex variable, and the path of integration is taken to be a closed contour in the plane of $u$ [to be replaced by the appropriate Riemann surface if $\phi(u)$ is non-uniform in this plane] enclosing no zero of $\phi(u)$ except $T$. In the same way $\int \frac{f_{1}^{\prime}(u) d u}{\phi(u)}, \int \frac{f_{2}^{\prime}(u) d u}{\phi(u)}, \int \frac{f_{3}^{\prime}(u) d u}{\phi(u)}$, or functions derived from them by differentiations, are all solutions of $\nabla^{2} U=V^{-2} \partial^{2} U / \partial t^{2}$. We may also notice that

$$
\frac{\partial}{\partial t} \int \frac{d u}{\phi(u)}=\int \frac{\partial}{\partial t} \frac{1}{\phi(u)} d u=-\frac{\partial}{\partial x} \int \frac{f_{1}^{\prime}(u)}{\phi(u)} d u-\frac{\partial}{\partial y} \int \frac{f_{2}^{\prime}(u)}{\phi(u)} d u-\frac{\partial}{\partial z} \int \frac{f_{s}^{\prime}(u)}{\phi(u)} d u .
$$

Hence

$$
\begin{aligned}
\frac{\partial}{\partial x} ! \frac{\partial}{\partial x} \int \frac{d u}{\phi(u)}+V^{-2} \frac{\partial}{\partial t} \int \frac{f_{1}^{\prime}(u)}{\phi(u)} d u ! & +\frac{\partial}{\partial y}\left\{\frac{\partial}{\partial y} \int \frac{d u}{\phi(u)}+V^{-2} \frac{\partial}{\partial t} \int \frac{f_{2}^{\prime}(u)}{\phi(u)} d u\right\} \\
& +\frac{\partial}{\partial z}\left\{\frac{\partial}{\partial z} \int \frac{d u}{\phi(u)}+V^{-2} \frac{\partial}{\partial t} \int \frac{f_{3}^{\prime}(u)}{\phi(u)} d u\right\}=0 .
\end{aligned}
$$

We shall now consider the electric force given by

$$
\left.\begin{array}{l}
K X=\frac{\partial}{\partial x} \int \frac{i e V d u}{\pi \phi(u)}+V^{-1} \frac{\partial}{\partial t} \int \frac{i e f_{1}^{\prime}(u) d u}{\pi \phi(u)}, \\
K Y=\frac{\partial}{\partial y} \int \frac{i e V d u}{\pi \phi(u)}+V^{-1} \frac{\partial}{\partial t} \int \frac{i e f_{2}^{\prime}(u) d u}{\pi \phi(u)}, \\
K Z=\frac{\partial}{\partial z} \int \frac{i e V d u}{\pi \phi(u)}+V^{-1} \frac{\partial}{\partial t} \int \frac{i e f_{3}^{\prime}(u) d u}{\pi \phi(u)},
\end{array}\right\}
$$

where $e$ is a constant and $i=\sqrt{-1}$.

From what has been proved above they are all solutions of

$$
\nabla^{2} U=V^{-2} \frac{\partial^{2} U}{\partial t^{2}}
$$

and, by (1), they satisfy $\partial X / \partial x+\partial Y / \partial y+\partial Z / \partial z=0$.

If $t>T>0$, the electric force at any point of the sphere

$$
\left[x-f_{1}(T)\right]^{2}+\left[y-f_{2}(T)\right]^{2}+\left[z-f_{3}(T)\right]^{2}=V^{2}(t-T)^{2}
$$


is given by

$$
\left.\begin{array}{l}
K X=-2 e V \frac{\partial}{\partial x} \frac{1}{\phi^{\prime}(T)}-2 e V^{-1} \frac{\partial}{\partial t} \frac{f_{1}^{\prime}(T)}{\phi^{\prime}(T)} \\
K Y=-2 e V \frac{\partial}{\partial y} \frac{1}{\phi^{\prime}(T)}-2 e V^{-1} \frac{\partial}{\partial t} \frac{f_{2}^{\prime}(T)}{\phi^{\prime}(T)}, \\
K Z=-2 e V \frac{\partial}{\partial z} \frac{1}{\phi^{\prime}(T)}-2 e V^{-1} \frac{\partial}{\partial t} \frac{f_{3}^{\prime}(T)}{\phi^{\prime}(T)} \cdot
\end{array}\right\}
$$

$X, Y$, and $Z$ become infinite only when $\phi^{\prime}(T)=0$, which, as we have seen, can take place only when $x=f_{1}(t), y=f_{2}(t), z=f_{3}(t)$. Then they become infinite to the same order as the components of force due to a point charge at rest. Hence the above expressions represent the field due to a simple singularity moving through the æther in a general manner, its position at a time $t$ being given by the co-ordinates $f_{1}(t), f_{2}(t), f_{3}(t)$. It remains to be shown that the strength of the charge associated with the electron remains constant. This we shall prove by evaluating the surface integral of the normal induction over the sphere $\phi(T)=0$. Remembering that $T$ is a function of $x, y, z$, and $t$, we get

$$
\begin{aligned}
& \iint K(l X+m Y+n Z) d S \\
& =4 e\left[V^{2}-\left\{f_{1}^{\prime}(T)\right\}^{2}-\left\{f_{2}^{\prime}(T)\right\}^{2}-\left\{f_{3}^{\prime}(T)\right\}^{2}\right] \iint \frac{d S}{\left[\phi^{\prime}(T)\right]^{2}} \\
& =2 \pi e\left[V^{2}-\left\{f_{1}^{\prime}(T)\right\}^{2}-\left\{f_{2}^{\prime}(T)\right\}^{2}-\left\{f_{3}^{\prime}(T)\right\}^{2}\right] \\
& \quad \times \int_{-V(t-T)}^{V(t-T)}\left[\xi \sqrt{\left.\left\{f_{1}^{\prime}(T)\right\}^{2}+\left\{f_{2}^{\prime}(T)\right\}^{2}+\left\{f_{3}^{\prime}(\bar{T})\right\}^{2}-V^{2}(t-T)\right]^{-2} d \xi=4 \pi e .}\right.
\end{aligned}
$$

Hence the strength of the electron is constant and equal to $e$.

From a physical point of view the above analysis shows that the state of the medium at any point depends only on the position and motion of the electron at a time $(t-T)$ previously where $T$ is the real root lying between 0 and $t$ of the equation

$$
\left[x-f_{1}(T)\right]^{2}+\left[y-f_{2}(T)\right]^{2}+\left[z-f_{3}(T)\right]^{2}=V^{2}(t-T)^{2} .
$$

The electron takes a time $T$ to get from its initial position to the point $f_{1}(T), f_{2}(T), f_{8}(T)$, and it is then producing an æthereal disturbance which after a further time $(t-T)$ reaches every point on the sphere $\phi(T)=0$. The fact that this sphere is of radius $V(t-T)$ is a direct proof of the fact that these disturbances travel with velocity $V$.

The condition $\left\{f_{1}^{\prime}(t)\right\}^{2}+\left\{f_{2}^{\prime}(t)\right\}^{2}+\left\{f_{3}^{\prime}(t)\right\}^{2}<V^{2}$ means, of course, that the velocity of the electron is less than the velocity of radiation. The theorem proved above that $T$ was a unique zero of $\phi(u)=0$ shows that 
there is only one position of the electron which is responsible for the disturbance at any point at any time. Our results also, from their method of investigation, can be applied only to points inside the sphere $\phi(0)=0$. What goes on outside this can, of course, be calculated from a knowledge of the values of the functions $f_{1}(t), f_{2}(t), f_{3}(t)$ for negative values of $t$.

The components of electric force can be written in the form

$$
K X=-\frac{\partial \psi}{\partial x}-\frac{\partial F}{\partial t}, \quad K Y=-\frac{\partial \psi}{\partial y}-\frac{\partial G}{\partial t}, \quad K Z=-\frac{\partial \psi}{\partial z}-\frac{\partial H}{\partial t},
$$

where

$$
\psi=\frac{2 e V}{\phi^{\prime}\left(T^{\prime}\right)}, \quad F=\frac{2 e}{V} \frac{f_{1}^{\prime}(T)}{\phi^{\prime}(T)}, \quad \ldots
$$

We can, hence, easily deduce the components of the magnetic force thus :-

$$
\iota=V^{2}\left(\frac{\partial H}{\partial y}-\frac{\partial G}{\partial z}\right)=2 e V\left(\frac{\partial}{\partial y} \frac{f_{3}^{\prime}(T)}{\phi^{\prime}(T)}-\frac{\partial}{\partial z} \frac{f_{2}^{\prime}(T)}{\phi^{\prime}(T)}\right) .
$$

It may be noticed that the expressions given above depend on both the velocity and the acceleration of the electron. Hence the expressions become discontinuous over a certain sphere $\phi(t)=0$ if the acceleration at the time $t$ becomes discontinuous: for instance, if the electron which has been moving with a uniform velocity begins to move with an acceleration, the sphere $\phi(t)=0$, every point of which is moving outwards with velocity $V$, is then a surface of discontinuity. The necessary conditions which must be satisfied at such a surface, if it be free from electrical charge, are (a) that the normal electric force must be continuous, and (b) that the difference betrreen the tangential components of the electrical force should be equal to the vector product of the difference of the tangential components of the magnetic induction and the normal velocity of the surface." For the components given above it will be found that both these conditions are fulfilled. In fact the electric force given by (3) has the component of the electric force normal to the sphere $\phi(T)=0$ independent of the acceleration, whilst the tangential component of the electric force is at right angles to the tangential component of the magnetic induction and equal to it multiplied by $V$, the directions being related to one another in the manner required by condition $(b)$.

The equations (2), (3), and (5) give then a complete solution for the field due to a singularity moving in any continuous manner. In the form (3) and (5) they are suitable for numerical calculation; in any given case it is only necessary to calculate a root of a given equation. For the explicit algebraical expression of our results it is necessary to be able to solve the equation $\phi(T)=0$. We can do this completely if the electron 
is at rest or moving with uniform velocity. We can also solve the case of an electron moving with a uniform acceleration. To do so we should require the solution of a biquadratic equation, and the results would be too complicated to be of any advantage. There are a few other cases in which the field of force can be obtained at particular points. We now proceed to consider these examples.

For the case of an electron at rest at the point $\left(x^{\prime}, y^{\prime}, z^{\prime}\right)$, the equation $\phi(T)=0$ becomes $\left(x-x^{\prime}\right)^{2}+\left(y-y^{\prime}\right)^{2}+\left(z-z^{\prime}\right)^{2}=V^{2}(t-T)^{2}$; so that

and

$$
\begin{aligned}
T & =t-V^{-1} \sqrt{ }\left(x-x^{\prime}\right)^{2}+\left(y-\overline{\left.y^{\prime}\right)^{2}+\left(z-z^{\prime}\right)^{2}}\right. \\
\phi^{\prime}(T) & =2 V^{2}(t-T)=2 V \sqrt{ }\left(x-x^{\prime}\right)^{2}+\overline{\left(y-y^{\prime}\right)^{2}+\left(z-z^{\prime}\right)^{2}} ;
\end{aligned}
$$

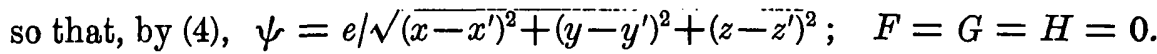

For the case of a point charge moving along the axis of $z$ with uniform velocity $v$, the equation $\phi(T)=0$ becomes $x^{2}+y^{2}+(z-v T)^{2}=V^{2}(t-T)^{2}$; so that

$$
T=\frac{-v z+V^{2} t-\sqrt{ }\left(V^{2}-v^{2}\right)\left(x^{2}+y^{2}\right)+V^{2}(z-v t)^{2}}{V^{2}-v^{2}},
$$

giving

$$
\psi=\frac{e V}{\left\{\left(V^{2}-v^{2}\right)\left(x^{2}+y^{2}\right)+V^{2}(z-v t)^{2}\right\}^{2}}
$$

and

$$
F=G=0 ; \quad H=\frac{v e V}{\left\{\left(V^{2}-v^{2}\right)\left(x^{2}+y^{2}\right)+V^{2}(z-v t)^{2}\right\}^{\frac{1}{2}}},
$$

which lead to the well known solutions for this case.*

If we have an electron moving in a circle, we can find the forces at any point of the axis. For let the position of the electron be denoted by the coordinates $f_{1}(t), f_{2}(t), 0$ where $\left\{f_{1}(t)\right\}^{2}+\left\{f_{2}(t)\right\}^{2}=R^{2}$, a constant; then for any point $(0,0, z)$ on the axis of $z$ we have

$$
\phi(T) \equiv\left\{f_{1}(T)\right\}^{2}+\left\{f_{2}(T)\right\}^{2}+z^{2}-V^{2}(t-T)^{2}=0
$$

or $R^{2}+z^{2}-V^{2}(t-T)^{2}=0$, so that $T=t-\frac{\sqrt{R^{2}+z^{2}}}{V}$, giving

$$
\begin{aligned}
& K X=-\frac{e f_{1}(T)}{\left(R^{2}+z^{2}\right)^{\frac{2}{3}}}-\frac{e}{V^{2}\left(R^{2}+z^{2}\right)}\left[\sqrt{R^{2}} \overline{z^{2}} f_{1}^{\prime \prime}(T)+V f_{1}^{\prime}(T)\right], \\
& K Y=-\frac{e f_{2}(T)}{\left(R^{2}+z^{2}\right)^{\frac{3}{2}}}-\frac{e}{V^{2}\left(R^{2}+z^{2}\right)}\left[\sqrt{R^{2}+z^{2}} f_{2}^{\prime \prime}(T)+V f_{2}^{\prime}(T)\right], \\
& K Z=\frac{e z}{\left(R^{2}+z^{2}\right)^{\frac{1}{2}}},
\end{aligned}
$$




$$
\begin{aligned}
& \alpha=\frac{e z}{V\left(R^{2}+z^{2}\right)^{3}}\left[\sqrt{R^{2}+z^{2}} f_{2}^{\prime \prime}(T)+V f_{2}^{\prime}(T)\right], \\
& \beta=\frac{e z}{V\left(R^{2}+z^{2}\right)^{\frac{1}{2}}}\left[\sqrt{R^{2}+z^{2}} f_{1}^{\prime \prime}(T)+V f_{1}^{\prime}(T)\right], \\
& \begin{aligned}
\gamma=\frac{e}{V\left(R^{2}+z^{2}\right)^{3}}\left[R\left\{f_{1}(T) f_{2}^{\prime \prime}(T)-f_{2}(T) f_{1}^{\prime \prime}(T)\right\}\right. \\
\left.\quad+V\left\{f_{1}(T) f_{2}^{\prime}(T)-f_{2}(T) f_{1}^{\prime}(T)\right\}\right] .
\end{aligned}
\end{aligned}
$$

More generally we can find the force at any point of the axis of a cylinder if an electron is moving on its surface, the velocity resolved along the axis being constant. A similar investigation gives the forces at the centre of a sphere due to an electron moving in any manner on its surface.

If we consider an electron of strength $\frac{1}{2} e$ at the point $\left[f_{1}(t), f_{2}(t), f_{3}(t)\right]$ in conjunction with another of strength $-\frac{1}{2} e$ at the point $\left[-f_{1}(t),-f_{2}(t)\right.$, $\left.-f_{3}(t)\right]$, we find, on the supposition that the squares and products of $f_{1}(t)$, $f_{1}^{\prime}(t), \ldots$, may be neglected,

$$
\begin{aligned}
K X=e x\left\{\frac{x f_{1}^{\prime \prime}(T)+y f_{2}^{\prime \prime}(T)+z f_{3}^{\prime \prime}(T)}{V^{2} r^{3}}-\frac{3}{V} \frac{x f_{1}^{\prime}(T)+y f_{2}^{\prime}(T)+z f_{3}^{\prime}(T)}{r^{4}}\right. \\
\left.+3 \frac{x f_{1}(T)+y f_{2}(T)+z f_{3}(T)}{r^{5}}\right\}-\frac{e f_{1}(T)}{r^{3}}+\frac{e f_{1}^{\prime}(T)}{V r^{2}}-\frac{e f_{1}^{\prime \prime}(T)}{V^{2} r},
\end{aligned}
$$

where

$$
T=t-\frac{\sqrt{x^{2}+y^{2}+z^{2}}}{V}
$$

with similar values for $Y$ and $Z$. This gives us the solution for various kinds of vibrators of the Hertzian type by taking the moment to be represented by the vector $e f_{1}(T), e f_{2}(T), e f_{3}(T) .^{*}$

In all the above analysis the method consisted in making a contour integration about a certain zero of the function $\phi(u)$, so that the solutions were singular only at one point, and the medium contained the past history of the motion of the electron, the disturbance being propagated in diverging waves. It is interesting to observe that, if by an extension of our analysis we had selected in place of the zero $T$ the real zero which is greater than $t$, we should then have obtained solutions infinite only at one point, which is the point of convergence of the disturbances, and the medium now contains the future history of the motion of the electron. For the case of an electron at rest or in uniform motion, these solutions, as we might expect, are not different from one another.

- Cf. Macdonald, Electric Waves, p. 176. For the reverse process-passing from the solutions of Hertz to the case of an electron vibrating over a small path-see Larmor, Aither and Matter, pp. 221 et seq.; Righi, Archives Néerlandaises, Ser. 2, Tome v., p. 348. 


\section{On Moving Distributions of Electricity.}

If we have any distribution of electrons which on the average amount to a continuous volume, surface, or line distribution of electric charge, we can calculate the resulting field of force from a scalar potential

$$
\psi=\int \frac{2 V d m}{\phi^{\prime}(T)},
$$

where $d m$ is an element of charge, and from a vector potential $(F, G, H)$, where

$$
F=\int \frac{2 f_{1}^{\prime}(T)}{V \phi^{\prime}(T)} d m, \ldots,
$$

after the manner of (4) and (5).

As a single example we consider the case of a continuous stream of electrons amounting to a constant line density $\lambda$ travelling uniformly in a circle. We can then take

$$
\begin{aligned}
f_{1}(t) & =a \cos (p t+\theta), & f_{2}(t) & =a \sin (p t+\theta), \\
f_{3}(t) & =0, & d m & =a \lambda d \theta .
\end{aligned}
$$

In evaluating $\psi$ it will be better, instead of using $2 / \phi^{\prime}(T)$, to replace it by the complex integral used in (2), viz., $\int i e V \pi^{-1}\{\phi(u)\}^{-1} d u$. In this case

$$
\begin{aligned}
\phi(u) & \equiv\left[x-f_{1}(u)\right]^{2}+\left[y-f_{2}(u)\right]^{2}+\left[z-f_{3}(u)\right]^{2}-V^{2}(t-u)^{2} \\
& \equiv x^{2}+y^{2}+z^{2}+a^{2}-V^{2}(t-u)^{2}-2 x a \cos (p u+\theta)-2 y a \sin (p u+\theta) .
\end{aligned}
$$

Hence

$$
\psi=-\int d m \int \frac{i V}{\pi} \frac{d u}{\phi(u)} .
$$

The complex integral is supposed to be taken over a contour which encloses a special zero $T$ of $\phi(u)$. These special zeros of $\phi(u)$ for all values of $\theta$ are, from their nature, confined to the part of the axis of real quantities lying between 0 and $t$. If, then, we suppose the path of integration to enclose this region, we are able to change the order of the operations on the right-hand side of $(6)$; hence

$$
\psi=-\frac{i V}{\pi} \int d u \int_{0}^{2 \pi} \frac{a \lambda d \theta}{\phi(u)} .
$$

If we write $x^{2}+y^{2}+z^{2}=r^{2}, x^{2}+y^{2}=\boldsymbol{\varpi}^{2}$, we get

$$
\begin{aligned}
\int_{0}^{2 \pi} \frac{a \lambda d \theta}{\phi(u)} & =\int_{0}^{2 \pi} a \lambda d \theta\left\{r^{2}+a^{2}-V^{2}(t-u)^{2}-2 a x \cos (p u+\theta)\right. \\
& -2 a y \sin (p u+\theta)\}^{-1} \\
& =\pi a \lambda\left\{\left[r^{2}+a^{2}-V^{2}(t-u)^{2}\right]^{2}-4 \varpi^{2} a^{2}\right\}^{-1},
\end{aligned}
$$

so that

$$
\psi=-i V a \lambda \int d u\left\{\left[r^{2}+a^{2}-V^{2}(t-u)^{2}\right]^{2}-4 \varpi^{2} a^{2}\right\}^{-\frac{1}{2}}
$$

BRR. 2. vor. 1. No. 830 . 
or, changing to real variables, ${ }^{*}$

$$
=\int_{V\left(r^{2}+a^{2}-2 \pi a\right)}^{V\left(r^{2}+a^{2}+2 \pi a\right)} a \lambda d \xi\left\{\left[r^{2}+a^{2}-\zeta^{2}\right]^{2}-4 \varpi^{2} a^{2}\right\}^{-2} .
$$

Putting now $\xi^{2}=r^{2}+a^{2}-2 \varpi a \cos \phi$, we get

$$
\psi=\int_{0}^{\pi} \frac{a \lambda d \phi}{\left(r^{2}+a^{2}-2 \varpi a \cos \phi\right)^{\frac{2}{2}}}
$$

which is the ordinary electrostatic potential of a uniform ring at rest. In a similar manner we can show that the vector potential is the same as that due to a circular current of strength $p a \lambda$.

A solution of the equation $-V^{-2} \partial^{2} U / \partial t^{2}+\nabla^{2} U+4 \pi \rho=0$ appropriate for the time $t$ is $\iiint r^{\prime-1} \rho^{\prime} d \xi^{\prime} d \eta^{\prime} d \xi^{\prime}$, where

$$
r^{\prime 2}=\left(x-\xi^{\prime}\right)^{2}+\left(y-\eta^{\prime}\right)^{2}+\left(z-\xi^{\prime}\right)^{2}
$$

and $\rho^{\prime}$ denotes the value of $\rho$ at the point $\xi^{\prime}, \eta^{\prime}, \xi^{\prime}$, and the time $t-r^{\prime} / V$. Starting from this fact, the solutions for any moving distribution of matter have been given by Macdonald.t We shall now show the connection between the solutions given by him and those given above.

The position of any electron is a function of its initial position and of the time, whilst the density at any part is a function only of the initial position of that part. If, then, $\rho$ be the density at the point $\xi, \eta$, $\xi$, which was initially at the point $\xi_{0}, \eta_{0}, \xi_{0}$, we may write

$$
\xi=f_{1}\left(\xi_{0}, \eta_{0}, \xi_{0}, t\right), \quad \eta=f_{2}(\ldots), \quad \xi=f_{3}(\ldots), \quad \rho=F\left(\xi_{0}, \eta_{0}, \xi_{0}\right) \text {. }
$$

In the formula $\psi=\int\left[\phi^{\prime}(T)\right]^{-1} 2 V d m$ we also require $T$. This is defined by the following equations :-

$$
\xi^{\prime}=f_{1}\left(\xi_{0}, \eta_{0}, \xi_{0}, T\right), \ldots, \quad\left(x-\xi^{\prime}\right)^{2}+\left(y-\eta^{\prime}\right)^{2}+\left(z-\xi^{\prime}\right)^{2}=V^{2}(t-T)^{2} .
$$

Now $d m=\rho d \xi_{0} d \eta_{0} d \xi_{0}$, and it is required to transform to the coordinates $\xi^{\prime}, \eta^{\prime}, \xi^{\prime}$. By using such formulæ as

$$
\begin{aligned}
\frac{\partial \xi^{\prime}}{\partial \xi_{0}} & =\frac{\partial f_{1}}{\partial \xi_{0}}+\frac{\partial f_{1}}{\partial T} \frac{\partial T}{\partial \xi_{0}} \\
& =\frac{\partial f_{1}}{\partial \xi_{0}}+V^{-2}(t-T)^{-1} \frac{\partial f_{1}}{\partial T}\left\{\left(x-\xi^{\prime}\right) \frac{\partial \xi^{\prime}}{\partial \xi_{0}}+\left(y-\eta^{\prime}\right) \frac{\partial \eta^{\prime}}{\partial \xi_{0}}+\left(z-\xi^{\prime}\right) \frac{\partial \xi^{\prime}}{\partial \xi_{0}}\right\},
\end{aligned}
$$

it can be verified without difficulty that

$$
\partial\left(\hat{\xi}_{0}, \eta_{0}, \xi_{0}\right) / \partial\left(\xi^{\prime}, \eta^{\prime}, \xi^{\prime}\right)=\frac{1}{2} V^{-1}\left[\left(x-\xi^{\prime}\right)^{2}+\left(y-\eta^{\prime}\right)^{2}+\left(z-\xi^{\prime}\right)^{2}\right]^{-\underline{1}} \phi^{\prime}(T),
$$

* Cf. Forsyth, Theory of Functions, p. 193.

+ Electric Warcs, Appendix C. For other investigations from the same point of view, see papers by E. Wiechert, C. H. Wind, H. des Coudres, Archives Néerlandaises, Série 2, Tome v. ; T. Levi-Civita, Nuovo Cimento, Serie 4, Vol. vr.; M. Abraham, Annalen der Physik, January, 1903. 
and it follows that

$$
\int 2\left[\phi^{\prime}(T)\right]^{-1} V d m=\iiint \rho\left[\left(x-\xi^{\prime}\right)^{2}+\left(y-\eta^{\prime}\right)^{2}+\left(z-\xi^{\prime}\right)^{2}\right]^{-\frac{1}{2}} d \xi^{\prime} d \eta^{\prime} d \xi^{\prime},
$$

showing that the two solutions are identical. The difference between them is analogous to that between the two methods of establishing the fundamental equations of fluid motion known as the methods of Lagrange and of Euler. In the one method attention is directed to what goes on in volume elements fixed in the æther, and would be the most suitable for any theory which regards electricity as continuous. In the other method the motion of each corpuscle is followed and advantage is gained when we regard electricity as consisting of discrete electrons.

\section{On the Radiation from an Electron.}

If (in the notation of the preceding section) we consider the shell bounded by the spheres $\phi(T)=0$ and $\phi(T-d T)=0$, the energy in it consists of two parts: one becomes negligible when $t$ becomes large ( $d T$ remaining the same size); the other remains finite. This second part is the energy radiated, and when divided by $d T$ we get the rate at which energy is being radiated at the time $T$. We shall proceed to find this rate of waste for the case of an electron moving in any manner. The thickness of the shell at any point is

$$
\frac{1}{2} V^{-1} \phi^{\prime}(T) d T
$$

so that the required quantity is

$$
\frac{1}{16 \pi} \iint K V^{-1}\left[K\left(X^{2}+Y^{2}+Z^{2}\right)+\mu\left(\alpha^{2}+\beta^{2}+\gamma^{2}\right)\right] \phi^{\prime}(T) d S,
$$

the integration being taken over the sphere

$$
\left[x-f_{1}(T)\right]^{2}+\left[y-f_{2}(T)\right]^{2}+\left[z-f_{3}(T)\right]^{2}=V^{2}(t-T)^{2}
$$

and $t$ being made infinitely great. To simplify the integration we can take the point $\left[f_{1}(T), f_{2}(T), f_{3}(T)\right]$ to be origin and the axis of $z$ the direction $\left[f_{1}^{\prime}(T), f_{2}^{\prime}(T), f_{3}^{\prime}(T)\right]$. Then, putting

$$
v^{2}=\left[f_{1}^{\prime}(T)\right]^{2}+\left[f_{2}^{\prime}\left(T^{\prime}\right)\right]^{2}+\left[f_{3}^{\prime}(T)\right]^{2}, \quad a^{2}=\left[f_{1}^{\prime \prime}(T)\right]^{2}+\left[f_{2}^{\prime \prime}(T)\right]^{2}+\left[f_{s}^{\prime \prime}(T)\right]^{2},
$$

$\alpha=$ the angle between $v$ and $a$,

$$
d S=V^{2}(t-T)^{2} \sin \theta d \theta d \phi,
$$

$\theta$ being the angle made with the direction of the velocity and $\phi$ the azimuthal angle. 
On integrating with respect to $\phi$, we get

$\frac{e^{2} a^{2}}{4 K} \int_{0}^{\pi}\left\{-\left(V^{2}-v^{2}\right)\left(\sin ^{2} \alpha \sin ^{2} \theta+2 \cos ^{2} \theta \cos ^{2} \alpha\right)+4 v \cos ^{2} \alpha \cos \theta(V-v \cos \theta)\right.$

which is equal to

$$
\left.+2(V-v \cos \theta)^{2}\right\} \frac{\sin \theta d \theta}{(V-v \cos \theta)^{5}},
$$

$$
\frac{2}{3} \frac{V e^{2} a^{2}}{K} \frac{V^{2}-v^{2} \sin ^{2} a}{\left(V^{2}-v^{2}\right)^{8}} .
$$

If $v^{2}$ is negligible, this expression becomes $\frac{2}{3} e^{2} a^{2} / K V^{8}$ (or if $\mu=1$ ) $=\frac{2}{3} e^{2} a^{2} \mid V$, which agrees with the rate of radiation given by Larmor* for an electron moving slowly. When $v^{2}$ cannot be neglected the result agrees with that given by Heavisidet on writing $Q^{2} / 4 \pi$ instead of $e^{2}$.

\section{On the Solutions of the Equations when the Velocity is greater than or equal to that of Radiation.}

In this section we consider briefly what the solutions (2) of $\$ 1$ become when the velocity of the singularity is not less than $V$. The integration is supposed to extend around a contour enclosing any real zeros of $\phi(u)$ between 0 and $t$. We have then to commence by discussing the real zeros of $\phi(u)$ between these limits. If $a$ is a real zero of $\phi^{\prime}(u)=0$, then $\phi(a)$ will be negative, zero, or positive, according as the point $(x, y, z)$ is inside, on, or outside the surface which is got by eliminating $a$ between $\phi^{\prime}(a)=0$ and $\phi(a)=0$. This surface, which is the envelope of the sphere $\phi(u)=0$, was imaginary in $\$ 1$ with the exception of one point-the point at which the electron was. In this case it consists of a conical surface, the apex being the electron, and its end is closed by the sphere $\phi(0)=0$. Leaving out of consideration the case in which this surface meets itself or the sphere again, we see that space is divided into three parts: (A) inside the sphere whose equation is $\phi(0)=0$, (B) outside the sphere but inside the envelope, (C) outside both. In (A) $\phi(0)$ is negative, $\phi(t)$ positive, $\phi(a)$ negative. Hence there is one real zero of $\phi(u)=0$. In (B) $\phi(0)$ is positive, $\phi(t)$ is positive, and $\phi(a)$ is negative; hence there are two real zeros of $\phi(u)=0$. In (C) there is no real zero. That is to say, in (A) the state of the medium at any point is due to one position of the electron, but in (B) there are two positions. For example, if we take a singularity moving with uniform velocity $v$ along the axis of $z$, the envelope will be a right circular cone of semi-angle $\sin ^{-1} V / v$. In the region (B), i.e., in the cone, the integrals vanish; for they enclose all the zeros

- Ether and Matter, p. 227.

+ Nature, November 6th, 1902. 
of $\phi(u)$. Hence in this region there is no electric or magnetic force. Inside (A) the solutions are of the same form as when $v=V$, and on the boundary the solutions will be infinite.

When the velocity $=V$, the result of substituting a zero $a$ of $\phi^{\prime}(a)$ in $\phi(a)$ is in general positive, but it will be zero if

$$
\frac{x-f_{1}(a)}{f_{1}^{\prime}(a)}=\frac{y-f_{2}(a)}{f_{2}^{\prime}(a)}=\frac{z-f_{3}(a)}{f_{3}^{\prime}(a)} ;
$$

so that on any sphere $\dot{\phi}(u)=0$ there is one point at which $\phi^{\prime}(u)=0$. It is the point of the sphere to which the velocity at the time $u$ is directed. With the exceptions of these points (which lie on an involute of the curve along which the singularity moves), the solution (2) holds inside the sphere $\phi(0)=0$.

As to the singularities which occur in these solutions they must be regarded in the same way as those which are on a sphere due to the uniform motion in a straight line of an electron moving with the velocity of radiation, ${ }^{*}$ i.e., they are to be regarded as places in which the forces become infinitely great without giving any convergence.

- Heaviside, Electromaynetic Theory. 